\title{
Involvement of Oct 4 in the pathogenesis of thoracic aortic dissection via inducing the dedifferentiated phenotype of human aortic smooth muscle cells by directly upregulating KLF5
}

\author{
Yan Yan, MD, ${ }^{\mathrm{a}}$ Meng-Wei Tan, MD, ${ }^{\mathrm{a}}$ Xiang Xue, MD, ${ }^{\mathrm{a}}$ Xue-Yan Ding, MD, ${ }^{\mathrm{b}}$ Guo-Kun Wang, MD, ${ }^{\mathrm{a}}$ and \\ Zhi-Yun Xu, MD
}

\begin{abstract}
Objective: To investigate the expression of Oct 4 in human thoracic aortic dissection (TAD) and the regulation mechanisms of Oct 4 on phenotype transition of human aortic smooth muscle cells (HASMCs).

Methods: Aortic samples from TAD patients $(n=12)$ and organ donors $(n=6)$ were collected. qRT-PCR, western blot, and immunohistochemistry were performed to identify Oct4 expression in aortic media. Immunofluorescence was performed to analyze Oct 4 expression in primary HASMCs. Oct4A and Oct4B isoforms were detected. Gain-of-function experiments were performed to determine the effects of Oct4 on HASMC phenotype transition. Chromatin immunoprecipitation, luciferase assay, and rescue experiments were performed to analyze mechanisms of Oct4 on HASMC phenotype transition.
\end{abstract}

Results: Oct 4 expression levels, especially the Oct $4 \mathrm{~A}$ isoform, were significantly higher in TAD patients compared with normal controls. Notably, Oct 4 presented a strong and strict nuclear localization in primary HASMCs of TAD patients but a mild and diffuse distribution in both cytoplasm and nucleus in the control group. Overexpression of Oct 4 induced dedifferentiation of HASMCs characterized by decreased contractile proteins and elevated migration capability. Krüppel-like factor 5 (KLF5) was found to be a directly regulated target gene of Oct4 in HASMCs. Furthermore, downregulation of KLF5 significantly alleviated the effects of Oct 4 on phenotype transition of HASMCs.

Conclusions: Oct 4 expression was significantly upregulated in aortic tissues and primary HASMCs of TAD patients. The increased Oct 4 induced phenotype transition of HASMCs from the contractile type to the synthetic type by directly upregulating KLF5. (J Thorac Cardiovasc Surg 2016;152:820-9)

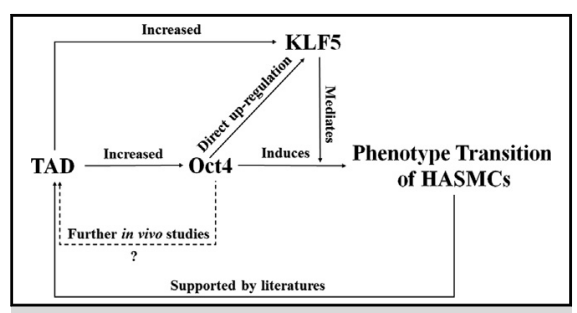

Involvement and mechanisms of Oct4 and KLF5 on HASMC phenotype transition in TAD.

\section{Central Message}

Increased Oct 4 in aortic media and primary HASMCs of TAD patients induces HASMC phenotype transition by directly upregulating KLF5.

\section{Perspective}

Elucidating the mechanisms of Oct4 on phenotype transition of human aortic smooth muscle cells from the contractile type to the synthetic type in the pathogenesis of thoracic aortic dissection might contribute to improvements in clinical treatment strategies of thoracic aortic dissection in the future.

See Editorial Commentary page 830.
Acute aortic dissection is a life-threatening disease with a reported annual incidence of $6 / 100,000$ and a 30-day in-hospital mortality of $47.4 \%{ }^{1}$ Various surgical strategies

\footnotetext{
From the ${ }^{\text {a Department }}$ of Cardiothoracic Surgery, Changhai Hospital, Second Military Medical University, Shanghai; and ${ }^{\mathrm{b}}$ Cardiovascular Therapeutic Centre, The 117 Hospital of the Chinese People's Liberation Army, Hangzhou, Zhejiang, China This work was supported by the National Natural Science Foundation of China (grant number 81300233 to M.-W.T.).

Y.Y., X.X., and X.-Y.D. contributed equally to this work.

Received for publication Jan 8, 2016; revisions received April 27, 2016; accepted for publication May 20, 2016; available ahead of print June 25, 2016.

Address for reprints: Meng-Wei Tan, MD, and Zhi-Yun Xu, MD, Department of Cardiothoracic Surgery, Changhai Hospital, Second Military Medical University, 168 Changhai Rd, Shanghai 200433, China (E-mail: tmengweismmu@163.com and xuzhiyunsmmu@163.com).

$0022-5223 / \$ 36.00$

Copyright (c) 2016 by The American Association for Thoracic Surgery

http://dx.doi.org/10.1016/j.jtcvs.2016.05.036
}

such as the frozen elephant trunk procedure and endovascular repair techniques have been introduced for its treatment. ${ }^{2,3}$ The pathogenesis of thoracic aortic dissection (TAD) has been extensively studied in recent years but still has not been thoroughly elucidated. Since in-depth knowledge on TAD pathogenesis may improve its clinical treatment strategy in the future, it is essential to further clarify the mechanisms of TAD. As the major cellular

Scanning this QR code will take you to the supplemental video, figures, and tables for the article. 

Abbreviations and Acronyms
ChIP = chromatin immunoprecipitation
ESCs = embryonic stem cells
HASMCs $=$ human aortic smooth muscle cells
KLF5 = Krüppel-like factor 5
Oct4 = Octamer binding protein-4
$\mathrm{TAD}=$ thoracic aortic dissection

components of aortic media and the primary source of extracellular matrix, the integrity of structures and functions of human aortic smooth muscle cells (HASMCs) is critical for maintaining the normal biomechanical properties of the aortic wall. ${ }^{4,5}$ Dysfunctions of HASMCs, however, have been reported to be related to a variety of aortic diseases. ${ }^{6,7}$ HASMCs possess some phenotypic plasticity and 2 corresponding phenotypes called the contractile and synthetic phenotypes. The contractile phenotype of HASMCs is characterized by the high expression levels of contractile proteins including $\alpha$ smooth muscle actin ( $\alpha$-SMA), smooth muscle myosin heavy chain, and smooth muscle $22 \alpha$ (SM22 $\alpha$ ), as well as relatively low rates of migration and extracellular matrix synthesis. On the contrary, synthetic HASMCs have low levels of contractile gene expression and increased rates of migration and extracellular matrix secretion. ${ }^{8}$ The contractile HASMCs are the predominant phenotype under physiologic conditions. Under pathologic stimulations, such as vascular injury or disease, however, the terminally differentiated contractile HASMCs switch to the dedifferentiated synthetic state.

With regard to TAD, the transition of the HASMC phenotype from the physiologic contractile type to the synthetic type has been reported to be the pivotal pathologic focus and an early event of thoracic aortic diseases. ${ }^{6,9,10}$ However, the precise molecular mechanisms within the processes of this HASMCs phenotype transition still have not been thoroughly illuminated.

Octamer binding protein-4 (Oct4), officially termed PitOct-Unc class 5 homeobox 1 (POU5f1), is a member of the POU transcription factor family that contains the POU domain and has the ability to bind an octamer consensus sequence. As a key regulator of self-renewal and pluripotency, Oct 4 is generally expressed in pluripotent cells. ${ }^{11}$ Transduction of Oct 4 together with another 3 transcription factors into primary fibroblasts can establish induced pluripotent stem cells. ${ }^{12}$ With regard to the involvement of Oct 4 in human diseases, Oct 4 has been detected in tissues of lung adenocarcinoma and has been shown to induce epithelial-mesenchymal transition, a process that contributes to the switch of tumor cells to a more malignant phenotype with a higher migration capability and stemness. ${ }^{13}$ However, with regard to the biological functions of Oct4 in vascular diseases, reports are extremely rare. One study by Firth et al, ${ }^{14}$ reported increased Oct4 expression in pulmonary artery smooth muscle cells from patients with idiopathic pulmonary arterial hypertension (IPAH). To our knowledge, the biological functions and related mechanisms of Oct4 in the aforementioned phenotype transition of HASMCs, and their involvement in the pathogenesis of TAD, has never been reported.

To obtain insight into the role of Oct4 in TAD pathogenesis, we designed experiments to investigate whether Oct4 was upregulated in the aortic tissues of TAD patients and its effects on the phenotypic switching of HASMCs.

\section{MATERIALS AND METHODS}

\section{Tissue Specimens}

Dissected ascending aortic specimens $(\mathrm{n}=12)$ were obtained from 12 patients with acute TAD classified as Stanford type A who underwent open surgical treatment in our department. Patients with connective tissue diseases, bicuspid aortic valve malformation, immunologic diseases, syphilis, and a family history of aortic diseases were excluded. Ascending aortic samples $(n=6)$ in the control group were acquired from 6 donors without vascular diseases ( 1 from a patient undergoing heart transplantation and 5 from autopsy samples). The general clinical characteristics of the patients are listed in Table E1. Full-thickness proximal ascending aortic tissues were harvested in both groups. For the immunohistochemical staining study, the specimens were fixed in $4 \%$ paraformaldehyde and embedded in paraffin. For western blot and qRT-PCR analysis, the intima and adventitia of aortic tissues were removed and the tunica media was snap-frozen and stored in liquid nitrogen. All study protocols were conducted according to the Declaration of Helsinki and approved by the Medical Ethics Committee of Changhai Hospital. All patients or donors' relatives provided informed consent.

\section{Isolation and Culture of HASMCs}

For cell gain-of-function and rescue experiments, purchased HASMCs (Cascade Biologics, Portland, Ore) were cultured in Medium 231 with its growth supplements (Cascade Biologics). Cells between passages 3 and 5 were used for the experiments in this section. HEK293 cells were cultured in DMEM supplemented with $10 \%$ fetal bovine serum (FBS).

For analysis of Oct 4 expression profiles in HASMCs from dissected aorta, primary HASMCs were isolated from 3 patients and 3 normal controls. The intima and adventitia of the aorta were carefully removed. Subsequently, the media of aortic tissues were cut into $4 \times 4 \mathrm{~mm}$ squares, which were then digested in $0.25 \%$ trypsin for 30 minutes at $37^{\circ} \mathrm{C}$. Explants were seeded onto the surface of a 24-well culture plate, with 1 to 2 explants per well. DMEM ( $300 \mu \mathrm{L})$ with $20 \%$ FBS was added to each well and replaced with fresh medium twice per week. The cells at passage 1 were used for the immunofluorescence study.

\section{Immunohistochemistry}

Immunohistochemistry was performed as described previously. ${ }^{15}$ The primary antibodies used were anti-Oct4 (1:100, ab19857; Abcam, Cambridge, Mass) and anti-Krüppel-like factor 5 (KLF5) (1:50, BA2804-2; Boster, Pleasanton, Calif).

\section{Immunofluorescence}

Paraformaldehyde-fixed HASMCs were first incubated with primary antibodies, followed by incubation with secondary antibodies. Nuclei were counterstained with DAPI. The primary antibodies used were 
anti-Oct4 antibody (1:100, ab19857; Abcam), anti- $\alpha$-SMA antibody (1:200, BM0002; Boster), and anti-myosin heavy chain 11 (MYH11) antibody (1:50, sc-6956; Santa Cruz Biotechnology, Dallas, Tex). The secondary antibodies used were Alexa Fluor 594-conjugated anti-rabbit IgG (1:400; Jackson ImmunoResearch, West Grove, Pa) and Alexa Fluor 498-conjugated anti-rabbit IgG (1:400; Jackson ImmunoResearch).

\section{Small Interfering RNA and Transfection}

Small interfering RNA (siRNA) against small interfering KLF5 (siKLF5) was purchased from GenePharma (Shanghai, China). The nontargeting scramble siRNA (siScramble) served as a negative control. HASMCs were transfected with siRNA using Lipofectamine 2000 (Invitrogen, Carlsbad, Calif) according to the manufacturer's instructions.

\section{Scratch Wound Assay}

In the gain-of-function experiments, HASMCs were transfected with either Ad.Oct 4 or Ad.GFP. In rescue experiments, after being transfected with Ad.Oct4, the HASMCs were transfected with siKLF5 or siScramble. A straight-line scratch was then gently made in the center of the cell monolayer. Images were captured at 0 and 24 hours after scratch.

\section{Western Blot}

Western blot was performed as described previously. ${ }^{15}$ The primary antibodies used were anti-Oct4 (1:1000, ab19857; Abcam), anti-KLF5 (1:500, BA2804-2; Boster), anti- $\alpha$-SMA antibody (1:500, BM0002; Boster), anti-MYH11 antibody (1:500, sc-6956; Santa Cruz), anti-MMP2 antibody (1:1000, BS1236; Bioworld Technology, St. Louis Park, Minn), and anti-MMP9 antibody (1:1000, ab38898; Abcam).

\section{Quantitative RT-PCR Analysis}

Total RNAs were extracted using the TRIzol reagent (Invitrogen). RNAs were reverse transcribed to cDNA using PrimeScript RT reagent Kit (TaKaRa Bio, Dalian, China). SYBR Premix EX Taq (TaKaRa Bio) was used for qRT-PCR performed on a LightCycler 480 Real-Time PCR System (Hoffmann-La Roche, Basel Switzerland). Primer sequences used for genes in this study are listed in Table E2.

\section{Luciferase Reporter Assay}

The luciferase reporter assay was performed as previous described, ${ }^{15}$ with modifications (Appendix E1).

\section{Chromatin Immunoprecipitation}

The chromatin immunoprecipitation (ChIP) assay was performed as previously described, ${ }^{15}$ with modifications (Appendix E1).

\section{Statistical Analysis}

Data are presented as means \pm standard deviation. Statistical analyses were performed with Student's $t$ test using SPSS version 19.0 (SPSS, Chicago, Ill). A $P$ value less than .05 is considered to be statistically significant.

\section{RESULTS \\ Oct4 Expression Is Increased in Aortic Tissues and HASMCs From TAD Patients and Is Associated With Phenotype Switch of HASMCs}

First, we analyzed the expression of Oct 4 in dissected aortas from TAD patients. Western blot showed that the protein level of Oct 4 was significantly increased in aortic tissues from the TAD group compared with normal aortic samples (by 1.55 -fold; Figure $1, A$ and $B$ ). qRT-PCR also showed that Oct4 mRNA was markedly increased in the TAD group (by 4.46-fold; Figure E1). Immunohistochemistry demonstrated that there was a significant upregulation of Oct4 expression and remarkable nuclear localization of Oct 4 in HASMCs within the aortic media of TAD patients (Figure 1, C).

To further clarify the difference in Oct 4 expression levels in HASMCs from TAD patients and in normal controls, we isolated primary HASMCs and performed immunofluorescence staining. As shown in Figure 1, D, HASMCs that migrated from aortic explants of normal controls displayed an elongated, triangle, or spindle-like shape and cell polarity, while HASMCs that migrated from dissected aortic explants of TAD patients presented an oval or polygon-like morphology. Cells cultured from both dissected and normal aorta showed positive immunostaining of smooth muscle markers including $\alpha$-SMA and MYH11 (Figure 1,E), which confirmed that the cultured primary cells were HASMCs. Notably, the expression levels of contractile proteins in HASMCs from TAD patients were significantly lower than those in HASMCs from normal controls. Furthermore, immunofluorescence revealed that HASMCs from TAD patients presented more prominent Oct4-positive stains (increased by 1.47 -fold), which were specifically localized in the nucleus of HASMCs. On the contrary, Oct4 was mildly expressed and diffusely distributed in both the cytoplasm and nucleus of HASMCs from normal controls (Figure 1, F). These results suggested that Oct4 might play an important role in the transcriptional regulation of some genes in HASMCs, which contributed to the pathogenesis of TAD.

The different Oct4 mRNA splicing variants can generate 2 Oct4 protein isoforms (Oct4A and Oct4B). ${ }^{16}$ It is generally acknowledged that only the Oct4A isoform functions as a transcription factor and sustains stemness properties. ${ }^{16}$ As a result, it is important to distinguish the expression levels of Oct4A from Oct4B. As demonstrated in Figure 2, $A$, we applied 4 different primer sets as previously reported ${ }^{14,16,17}$ to identify the respective expression levels of the 2 Oct 4 isoforms. Primer sets amplifying both isoforms had positive bands in both the TAD and normal groups (Figure 2, B, Oct4-1 and Oct4-2), with bands in the TAD group being more prominent. However, the 2 primer sets specific for Oct4A isoform exclusively produced abundant amplicons in the TAD group but almost no PCR products in the normal group (Figure 2, B, Oct4A-1 and Oct4A-2).

Interestingly, we further found that although normal primary HASMCs at passage 1 did not express Oct4A (Figure 2, $C$, Oct4A-1 and Oct4A-2) but merely mild Oct4B (Figure 2, $C$, Oct4-1 and Oct4-2), they expressed both of the 2 isoforms when the normal primary HASMCs were passaged to the 5 th generation (Figure 2, C). Given the fact that the conventional passage of primary vascular 


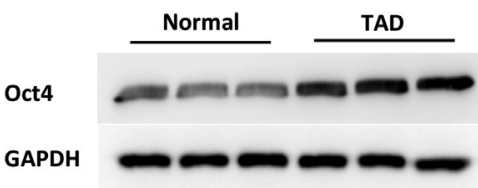

A
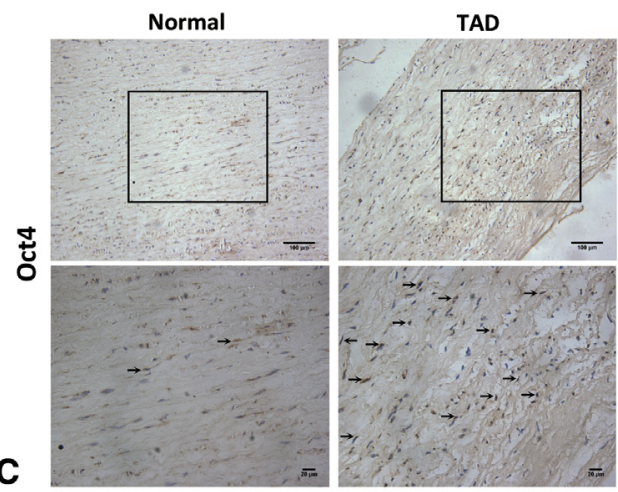

D

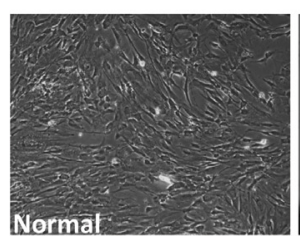

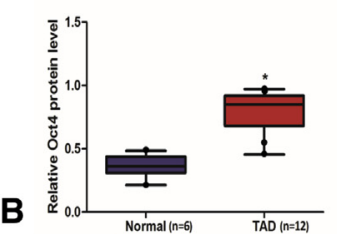

Normal (n=6) TAD $(n=12)$

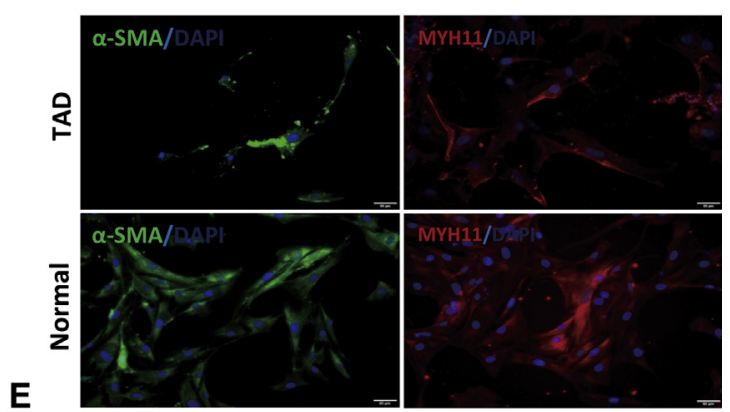

FIGURE 1. Increased Oct 4 expression in aortic tissues and HASMCs from TAD patients. A and B, Western blot showed that Oct4 protein in aortic tissues from TAD patients $(\mathrm{n}=12)$ was significantly higher than that in normal controls $(\mathrm{n}=6) * P<.05$ versus controls. $C$, Immunohistochemical staining showed that the expression of Oct4 was increased in the nucleus of HASMCs in aortic media from TAD patients. Arrows point to positive staining. D, Morphology of HASMCs that had migrated from aortic explants from TAD patients and normal controls. Magnification is $100 \times$. E, Immunofluorescent staining of $\alpha$-SMA (green) and MYH-11 (red) in HASMCs cultured from TAD tissues and normal aortas. Cells are counterstained with DAPI (blue). F, Immunofluorescent staining of Oct4 (red) in HASMCs cultured from TAD tissues and normal aortas, with insets of greater magnification. Oct4, Octamer binding protein-4; $G A P D H$, glyceraldehyde-3-phosphate dehydrogenase; TAD, thoracic aortic dissection; $\alpha$-SMA, $\alpha$-smooth muscle actin; MYH11, anti-myosin heavy chain 11.

smooth muscle cells can lead to phenotypic modulation, ${ }^{18,19}$ our results also imply that Oct4 might be involved in the phenotype switch of HASMCs during the pathologic processes of TAD.

\section{Oct4 Induced the Dedifferentiated Phenotype of}

\section{HASMCs and Upregulated its Migration Capability}

To further investigate whether Oct4 is involved in the phenotypic modulation of HASMCs, we examined the expression of Oct4 in synthetic HASMCs induced by tumor necrosis factor $\alpha$ (TNF- $\alpha)$ and found that stimulation of HASMCs with TNF- $\alpha$ resulted in the upregulation of both Oct 4 mRNA and protein (Figure E3). To clarify the potential biological functions of Oct4 on phenotypic switching of HASMCs, we transfected HASMCs with Ad.Oct4 and Ad.GFP. As shown in Figure 3, A, HASMCs could be efficiently transfected via our adenovirus vectors. Transduction of HASMCs with Ad.Oct4 significantly increased the expression of Oct4 mRNA and protein (Figure 3, $B$ and
C). Functionally, HASMCs transfected with Ad.Oct4 showed significantly reduced mRNA and protein levels of $\alpha$-SMA and MYH11 (Figure 3, $D$ and $E$ ), which were highly expressed in HASMCs of normal aorta. In addition, we also demonstrated that overexpression of Oct4 significantly increased the expression of MMP2 and MMP9 (Figure E4). Moreover, to investigate the impact of Oct4 on the migration capability of HASMCs, a scratch wound-healing assay was performed. It was demonstrated that the increased Oct4 expression significantly upregulated the migration capability of HASMCs (Figure $3, F$ and $G$ ).

\section{KLF5 Is the Target Gene of Oct4 in HASMCs With Elevated Expression in Aortic Tissues From TAD Patients, and KLF5 Promoter Can Be Activated by Oct4 Directly}

In order to elucidate the potential molecular mechanisms of Oct4 in the regulation of HASMCs phenotype transition, we performed a review of the literature to screen for 


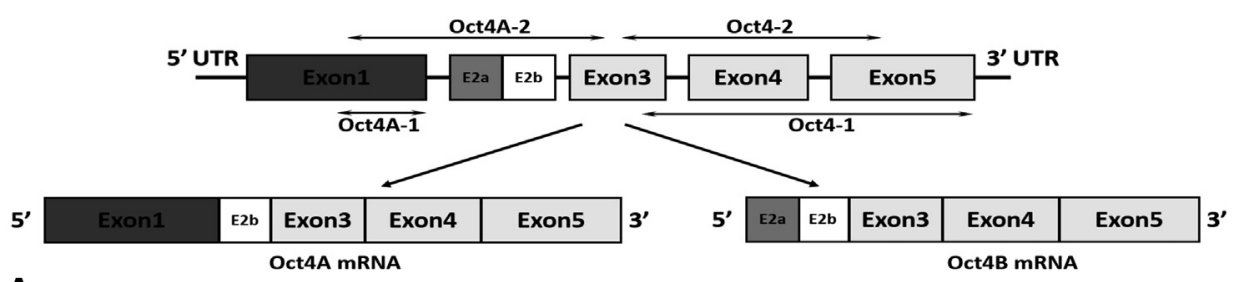

A
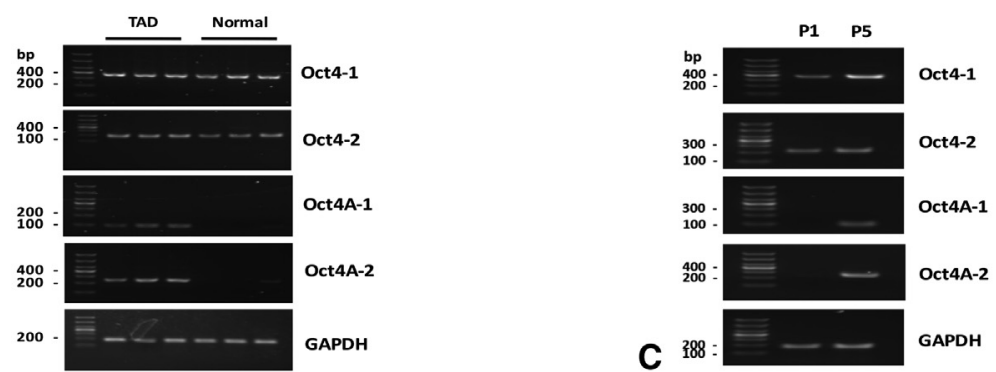

FIGURE 2. Analysis of expression of different Oct 4 isoforms in aortic tissues and HASMCs. A, Schematic diagram demonstrating the structure of human Oct4 transcripts. The regions amplified by corresponding primer sets used in our study are annotated by double-headed arrows. B, PCR results demonstrating expression levels of different Oct4 isoforms in aortic tissues indicated that primer sets amplifying both isoforms had positive results in both TAD and normal groups, with bands in the TAD group being more prominent. However, the 2 primer sets specific for the Oct $4 \mathrm{~A}$ isoform exclusively produced abundant amplicons in the TAD group, but almost no PCR products in the normal group. C, Representative images of PCR results in cultured HASMCs. All primers can produce positive bands in cells at P5, while almost no PCR products could be identified in cells at P1 using the 2 primer sets specific for Oct4A. Oct4, Octamer binding protein-4; TAD, thoracic aortic dissection; $P 1$, the first passage; P5, the 5th passage; GAPDH, glyceraldehyde-3-phosphate dehydrogenase.

A
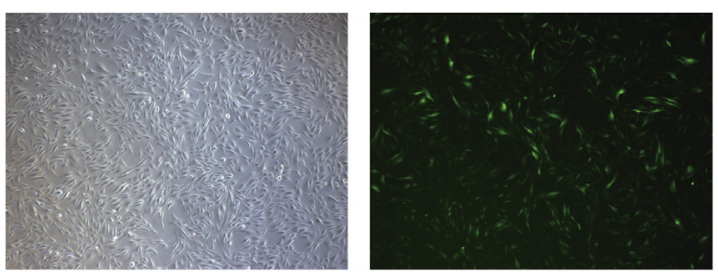

B

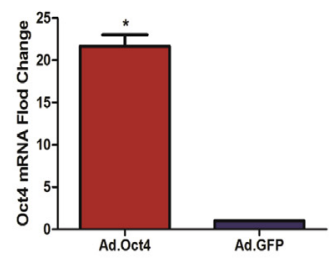

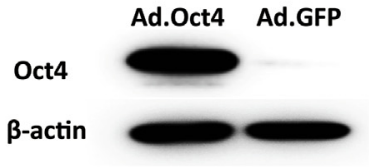

C

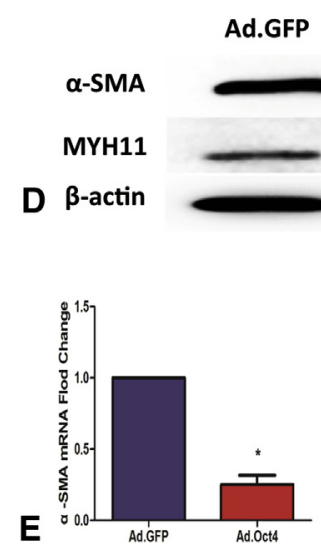

Ad.Oct4
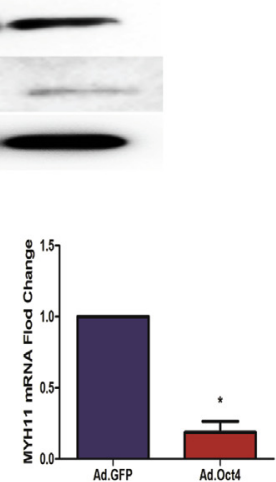
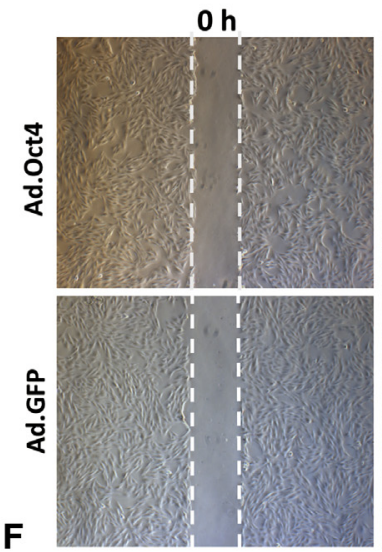

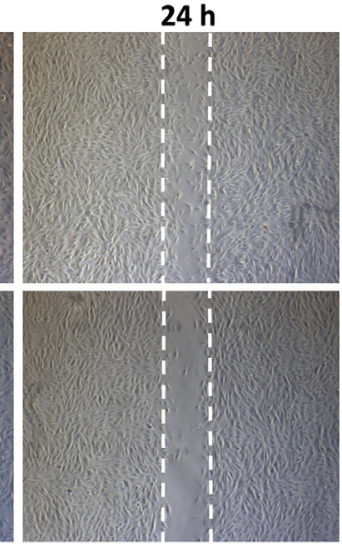

$24 \mathrm{~h}$

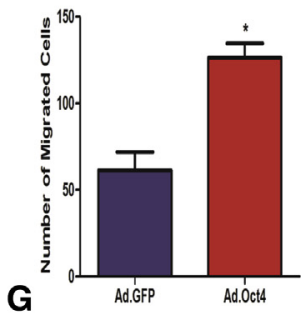

FIGURE 3. Oct 4 downregulated the expression of contractile proteins in HASMCs and increased their migration capability. A, The transfection efficiency of adenovirus vectors reached $\leq 90 \%$. B and C, qRT-PCR and western blot showed that the expression of Oct 4 was significantly increased in the Ad.Oct4 group compared with the Ad.GFP group ( $\mathrm{n}=3$ for each cell group). D and E, qRT-PCR and western blot showed that the expression of differentiated marker genes in HASMCs ( $\alpha$-SMA and MYH-11) significantly decreased in Ad.Oct4 HASMCs compared with Ad.GFP HASMCs ( $\mathrm{n}=3$ for each cell group). $* P<.05$ versus Ad.GFP HASMCs. F and G, Wound scratch assay results indicated that HASMCs transfected with Ad.Oct 4 had significantly higher migration ability compared with Ad.GFP HASMCs. Migrated cells were quantitated. The results are presented as means \pm standard deviation from 3 independent experiments performed in duplicate. ${ }^{*} P<.05$ versus Ad.GFP HASMCs. Ad.Oct4, Adenovirus overexpressing Oct4; Ad.GFP, adenovirus expressing GFP; Oct4, octamer binding protein-4; $\alpha$-SMA, $\alpha$-smooth muscle actin; MYH11, anti-myosin heavy chain 11 . 

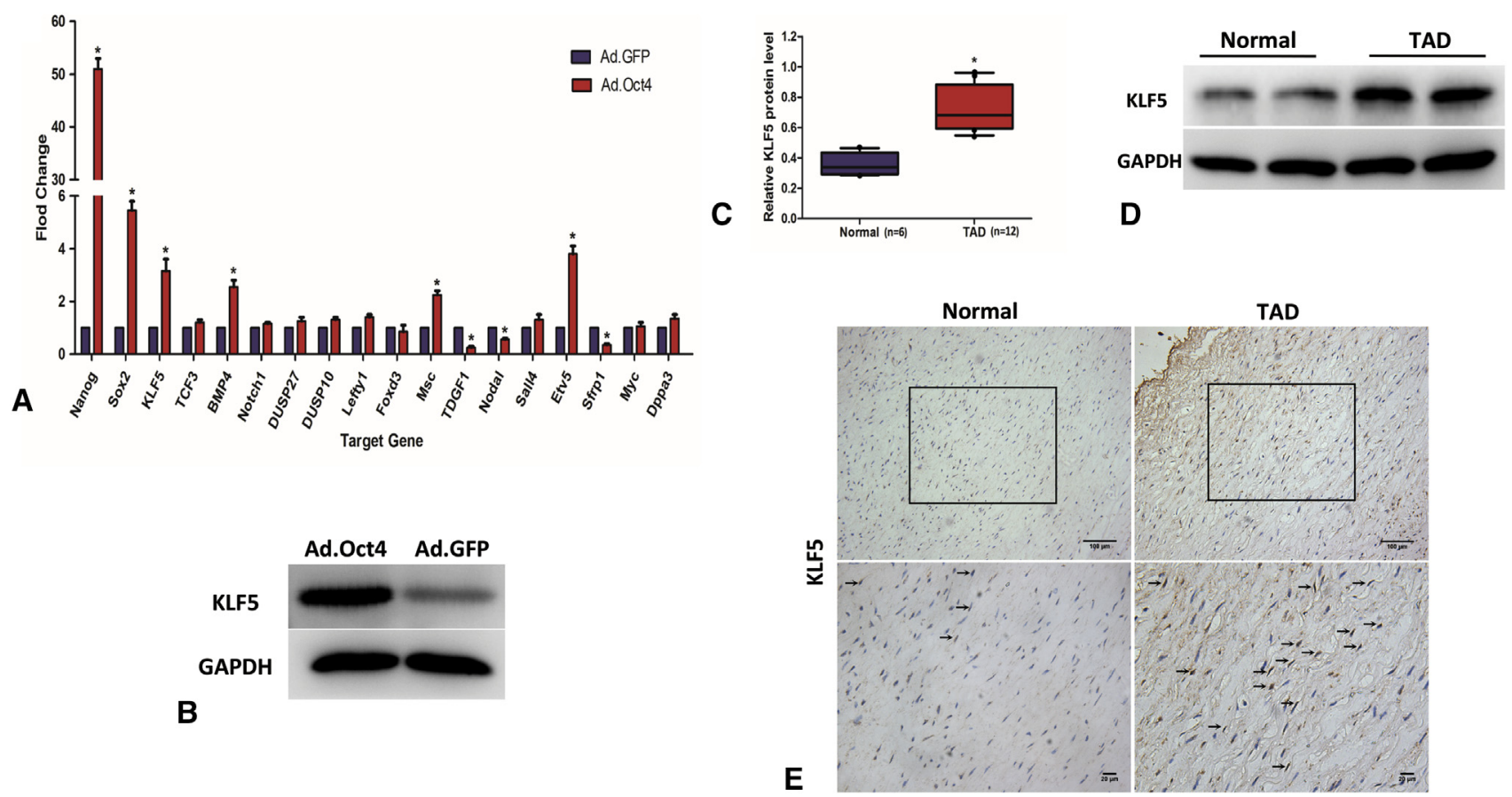

FIGURE 4. Identification of KLF5 as the target gene of Oct4 in HASMCs with increased expression in aortic tissues from TAD patients. A, qRT-PCR showed that the mRNA levels of KLF5, Nanog, Sox2, Etv5, and BMP4 were significantly upregulated in HASMCs transfected with Ad.Oct4 compared with those transfected with Ad.GFP ( $\mathrm{n}=3$ for each cell group). ${ }^{*} P<.05$ versus Ad.GFP HASMCs. B, Western blot showed that the protein levels of KLF5 in the Ad.Oct4 group were significantly higher than in the Ad.GFP group ( $\mathrm{n}=3$ for each cell group). C and D, The expressions levels of KLF5 protein were significantly higher in TAD patients $(\mathrm{n}=12)$ than in normal controls $(\mathrm{n}=6) . * P<.05$ versus normal aortas. E, Immunohistochemical staining showed that, compared with normal aorta, the KLF5 expression level increased in the nucleus of HASMCs within the aortic media of TAD patients. Arrows point to positive staining. KLF5, Krüppel-like factor 5; GAPDH, glyceraldehyde-3-phosphate dehydrogenase; Ad.Oct4, adenovirus overexpressing Oct4; Ad.GFP, adenovirus expressing GFP; TAD, thoracic aortic dissection.

candidate target genes of Oct4 that are related to cell differentiation and migration. ${ }^{20,21}$ qRT-PCR was performed to examine the expression levels of the selected 18 genes in HASMCs transfected with Ad.Oct4. It was demonstrated that, compared with the Ad.GFP group, overexpression of Oct4 in HASMCs significantly increased the mRNA levels of Nanog, Sox2, BMP4, Etv5, and KLF5, whereas the mRNA levels of TDGF1, Nodal, and Sfrp1 markedly decreased (Figure 4, A). Since KLF5 has been reported to be involved in the phenotype switch of vascular smooth muscle cells, ${ }^{22,23}$ we further confirmed the regulatory effects of Oct4 on KLF5 expression using western blot. It was documented that, compared with the Ad-GFP group, KLF5 was significantly upregulated in HASMCs transfected with Ad-Oct4 (Figure 4, B).

The expression of KLF5 in aortic tissues of TAD patients indicated that the protein level of KLF5 in the TAD group was significantly higher than that in the normal controls (Figure 4, $C$ and $D$ ). Immunohistochemistry revealed that KLF5 was highly expressed in the HASMCs of aortic media in TAD patients, but was rarely expressed in the aorta from normal controls.
To investigate whether Oct 4 directly regulates the expression of KLF5, we first predicted the potential binding sites of Oct 4 on the promoter sequence of KLF5 using the bioinformatics calculation tool PROMO 3.0. The 8-base-pair (bp) sequence (ATTTGCCT) starting at -903 bp upstream of the KLF5 transcription initiation site was predicted as a potential binding site of Oct4 (Figure 5, A). Subsequently, a ChIP assay was performed to confirm whether Oct4 was really recruited to this site. Using primer sets flanking a region containing the predicted candidate binding site, polymerase chain reaction produced an amplicon in the DNA immunoprecipitated with Oct4 antibody, which suggested that Oct4 could directly bind to the promoter of KLF5. Furthermore, we constructed a plasmid pGL3-Pro-WTKLF5 containing a wild-type KLF5 promoter and a motifmutant plasmid pGL3-Pro-MT-KLF5 in which the sequence of this predicted binding site was changed. These 2 plasmids were separately co-transfected with an Oct4 overexpression plasmid into 293 cells. Compared with the motif-mutant plasmid, co-transfection of Oct 4 significantly transactivated the luciferase reporter plasmid of pGL3-ProWT-KLF5 containing the wild-type KLF5 promoter 

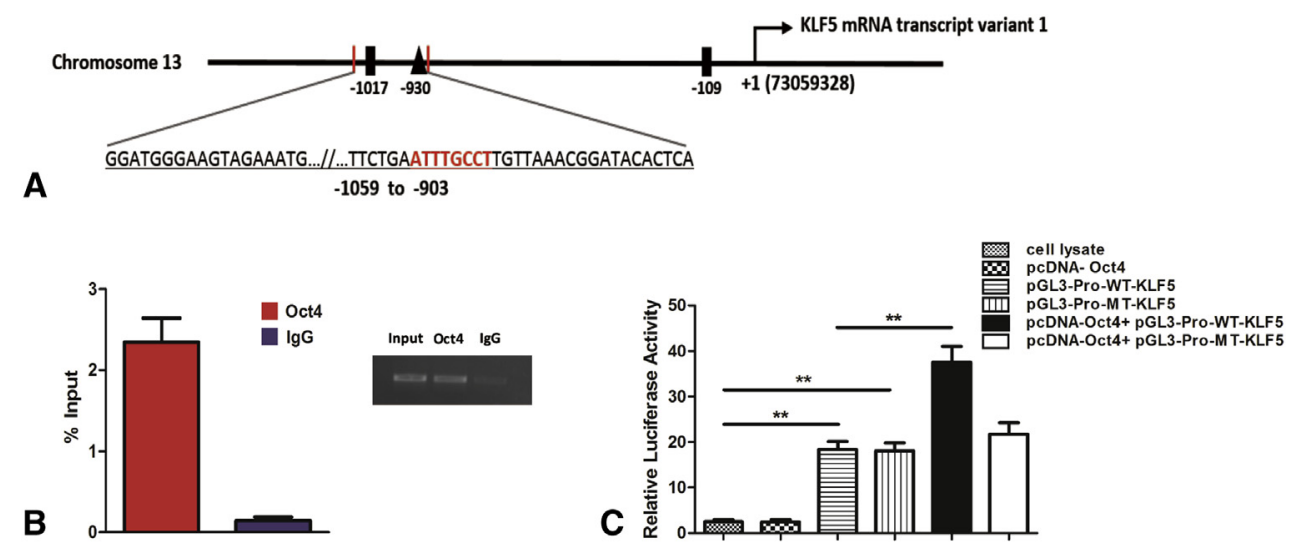

FIGURE 5. Oct4 activated KLF5 expression by directly binding to its promoter. A, Schematic of the KLF5 promoter with the Oct4 binding site depicted as triangle. The octamer motif bound by Oct 4 is highlighted with red letters. The positions of primer sets used for ChIP assay for the bound site are indicated by short red lines. The plasmid containing -109 to -1017 bp of the KLF5 promoter was constructed. B, After overexpression of Oct 4 in HASMCs, ChIP of Oct4 was performed. qRT-PCR was used to determine DNA enrichment in the KLF5 promoter relative to input. Normal rabbit IgG was used as a negative control. All experiments were performed in triplicate. C, The dual luciferase assays showed that Oct4 activated the promoter of pGL3-Pro-WT-KLF5 but had little effect on the activity of pGL3-Pro-MT-KLF5. The results are presented as means \pm standard deviation from 3 independent experiments. $* * P<.01$ versus controls. KLF5, Krüppel-like factor 5; Oct4, octamer binding protein-4.

(Figure 5, C). These results indicated that Oct 4 upregulated the KLF5 expression of HASMCs by directly binding to their promoter sequence and therefore increasing the transcription of KLF5.

\section{Oct4 Induced the Phenotype Transition of HASMCs by Upregulating KLF5 Expression}

Based on our aforementioned results, we further investigated whether KLF5 mediated these effects of Oct4 on the phenotypic switch of HASMCs. To substantiate this hypothesis, rescue experiments were performed by co-transfecting KLF5 siRNA (siKLF) and Ad.Oct4 into HASMCs as the experimental group. HASMCs co-transfected with siScramble and Ad.Oct4 served as the control. As shown in Figure 6, $A$, the transfection efficiency reached $\leq 90 \%$. In the Ad.Oct $4 / \mathrm{siKLF}$ group, the mRNA and protein levels of KLF5 were both significantly inhibited (Figure 6, $B$ and $C$ ). Functionally, Oct4 overexpression combined with KLF5 knockdown in the Ad.Oct $4 /$ siKLF group significantly resumed a relatively high expression level of the contractile proteins in HASMCs compared with the Ad.Oct4/siScramble group (Figure 6, $D$ and $E$ ). In addition, Oct4-induced upregulation of the migration capability of HASMCs, as shown in the Ad.Oct $4 /$ siScramble group, was drastically suppressed again when KLF5 was simultaneously knocked down (Figure 6, $F$ and $G$ ). These results indicated that the effects of Oct 4 on inducing the phenotypic switch of HASMCs from the contractile type to the synthetic type were at least partially mediated by its upregulation on KLF5 expression.

\section{DISCUSSION}

We found in the present study that Oct4, which was increased in the aortic media of TAD patients, induces the synthetic phenotype of HASMCs with accompanying increased migration capability by directly upregulating KLF5 expression. This is the first study to show the involvement and biological mechanisms of Oct4 in the phenotype transition of HASMCs and in the pathogenesis of TAD.

As a transcription factor essential for the pluripotency of embryonic stem cells (ESCs), Oct4 was believed to express mostly in adult germ cells under physiologic conditions. ${ }^{14}$ With regard to other somatic cells in adults, significant Oct4 expression and cell phenotypic switch have been observed in rat alveolar type I cells, which were traditionally considered to be a kind of terminally differentiated cells with no capability of phenotypic plasticity. ${ }^{24}$ However, with regard to pathologic status, the elevated expression levels of Oct 4 have been widely reported in series of cancer cell lines and tumor tissues such as bladder cancer and lung adenocarcinoma. ${ }^{11,13}$ With regard to the involvement of Oct4 in circulatory diseases, this unique study showed increased Oct4 expression in pulmonary smooth muscle cells of patients with IPAH. Our results with TAD tissues demonstrated for the first time that Oct4 is involved in the pathogenesis of TAD.

The human Oct 4 gene can generate 2 transcripts, Oct $4 \mathrm{~A}$ and Oct4B, as previously reported. It was definitively shown that only Oct $4 \mathrm{~A}$ possessed the capability of sustaining the pluripotency of cells. In our study, 4 different pairs of primer sets, 2 of which were specific for Oct $4 \mathrm{~A}$, 
A
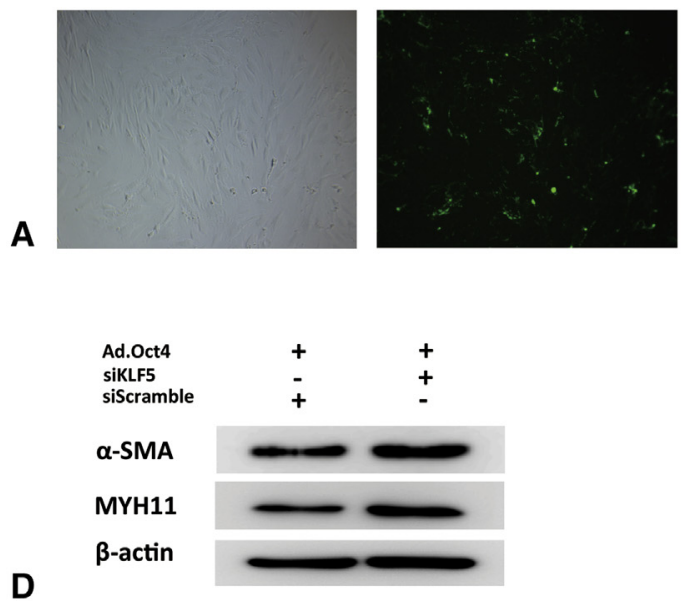

D
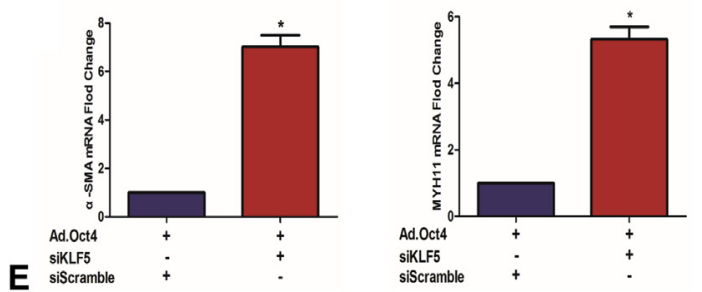
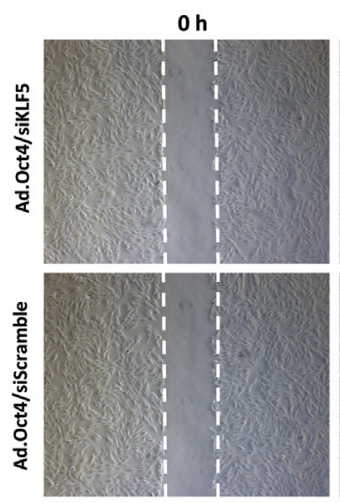

$\mathbf{F}$
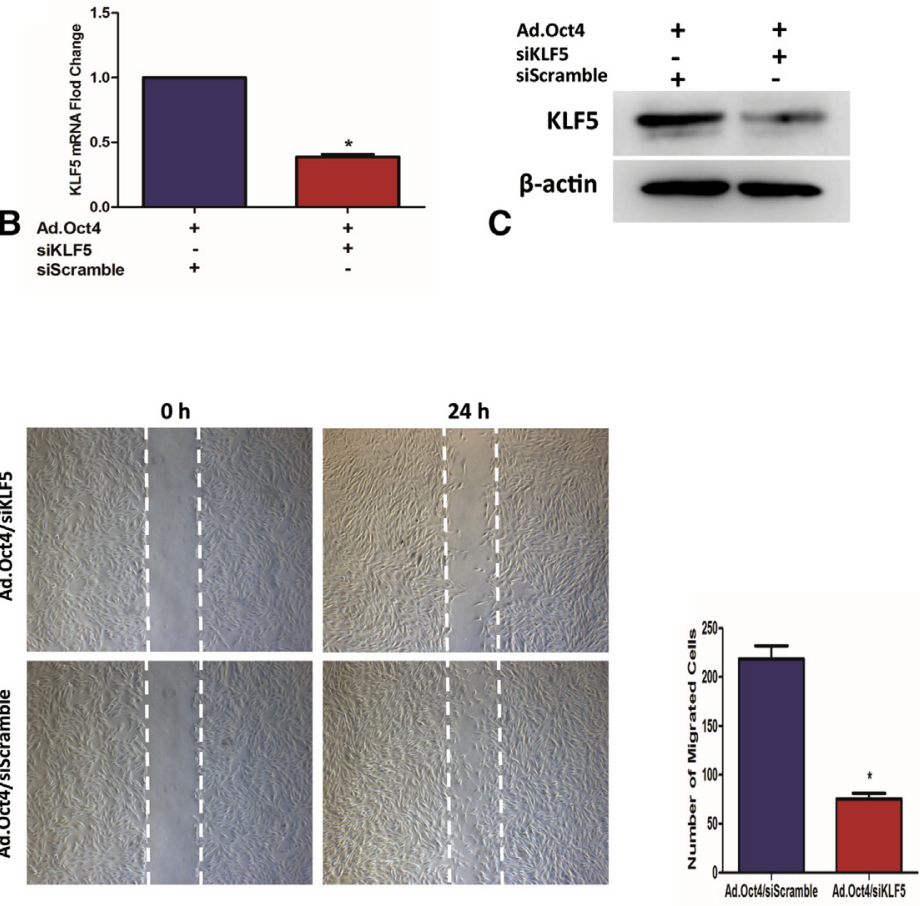

G

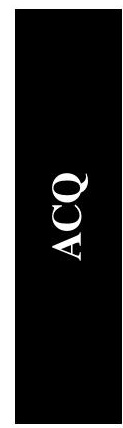

FIGURE 6. KLF5 mediated the inducement of Oct 4 on the phenotypic switching of HASMCs. A, HASMCs were transfected with FAM-siRNA against KLF. The transfection efficiency reached $\leq 90 \%$. B and C, qRT-PCR and western blot showed that the expression of KLF5 was significantly inhibited by siKLF5 ( $\mathrm{n}=3$ for each cell group). D and E, qRT-PCR and western blot showed that the mRNA and protein levels of $\alpha$-SMA and MYH-11 in HASMCs cotransfected with Ad.Oct4 and siKLF5 (Ad.Oct4/siKLF) were significantly higher compared with HASMCs co-transfected with Ad.Oct4 and siScramble (Ad.Oct4/siScramble) $(\mathrm{n}=3$ for each cell group). $* P<.05$ versus Ad.Oct4/siScramble. $\mathrm{F}$ and G, Wound scratch assay indicated that Ad.Oct4/siKLF5 HASMCs presented significantly lower migration ability. Migrated cells were quantitated. The results are presented as means \pm standard deviation from 3 independent experiments performed in duplicate. $* P<.05$ versus Ad.Oct4/siScramble. Ad.Oct4, Adenovirus overexpressing Oct4; siKLF5, small interfering RNA against KLF5; siScramble, scramble negative control siRNA; $\alpha$-SMA, $\alpha$-smooth muscle actin; MYH11, anti-myosin heavy chain 11; KLF5, Krüppel-like factor 5.

were used to elucidate the precise expression situation of the 2 Oct 4 isoforms in aortic tissues from TAD patients and controls. Our results indicated that only Oct4A was specifically expressed in the aorta of TAD patients. In the normal control group, primers specific for Oct4A barely generate any observable bands. Our results on the alternation of Oct4A expression after several passages of normal HASMCs implied indirectly that, as a stemness marker gene, Oct 4 might participate in the process of the phenotype transition of HASMCs because previous studies have verified that in vitro passages of primary vascular smooth muscle cells lead to a phenotypic switch characterized by dramatically reduced contractile proteins. ${ }^{18,19,25}$ In vitro functional experiments verified that Oct4 drastically downregulated the expression levels of $\alpha$-SMA, as well as MYH11 in HASMCs, and enhanced its migration capability.

The switch of the HASMCs phenotype from the terminally differentiated contractile type to the synthetic type, characterized by reduced expression of contractile proteins and increased migration, has been reported to play an essential role in the pathogenesis of TAD. ${ }^{6,26}$ In a recent study, Salmon et $\mathrm{al}^{9}$ reported that KLF4, a well-known transcription factor crucial for the pluripotency

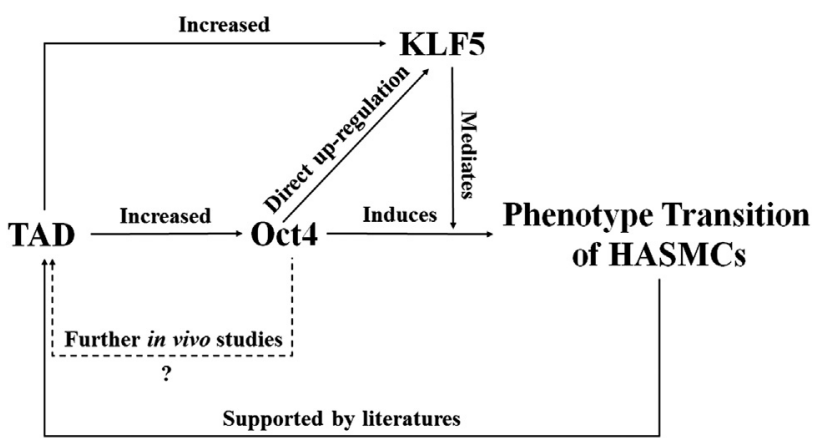

FIGURE 7. Expression of Oct 4 was significantly higher in TAD patients as compared with normal controls. Overexpression of Oct 4 induced a phenotype transition of HASMCs from the contractile type to the synthetic type by directly up-regulating KLF5, expression of which was increased as well in aortic media of TAD patients. Oct4, Octamer binding protein-4; $T A D$, thoracic aortic dissection; HASMCs, human aortic smooth muscle cells; KLF5, Krüppel-like factor 5 . 


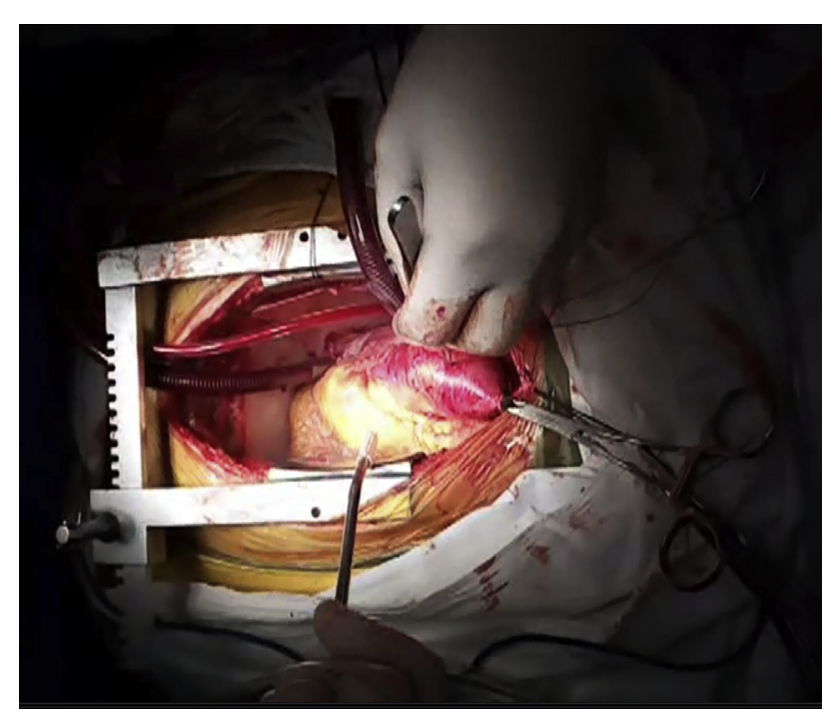

VIDEO 1. Bentall procedure combined with Sun's procedure for acute type A aortic dissection. Preoperative total aortic computed tomography angiography definitely diagnosed Stanford type A aortic dissection, with dissection from the ascending aorta to the abdominal aorta. Transthoracic echocardiography revealed severe aortic insufficiency. The procedure was carried out using a median sternotomy, and cannulation of the femoral artery was used for cardiopulmonary bypass. After the ascending aorta was clamped and opened, cardioplegia was perfused through the coronary ostia. The Bentall procedure was used for the treatment of the aortic root. A mechanical valve was continuously sewn to a 4-branch conduit to make a valved 4-branch conduit for the replacement of the aortic valve and reconstruction of the ascending aorta and aortic arch. The aortic valve and dissected ascending aorta were resected and the mechanical valve was sewn to the aortic annulus with interrupted sutures. Then, left and right coronary ostia were anastomosed to the valved graft. When the core temperature reached $26^{\circ} \mathrm{C}$, circulatory arrest was initiated, and antegrade cerebral perfusion was administered. Subsequently, a stent graft was inserted into the true lumen of the distal aorta through the arch incision. Then, the distal aorta incorporating the stent graft was anastomosed to the distal end of the 4-branch conduit, with antegrade perfusion of the lower body through the perfusion limb of the 4-branch graft. Finally, left carotid artery, innominate artery, and left subclavian artery were anastomosed to the corresponding arch limbs of the 4-branch graft. Video available at http://www.jtcvsonline.org/article/ S0022-5223(16)30474-3/addons.

in ESCs, also played an important role in the development of aortic aneurysm by promoting the phenotype switch of HASMCs. Similarly, our present findings suggest that Oct4, another well-known stemness-related transcription factor, was important in the regulation of the phenotype switch of HASMCs from the contractile type to the synthetic type, which was considered to be important in the development of TAD. ${ }^{6,26}$

KLF5, which was first found to be directly upregulated by Oct 4 in HASMCs in our study, is a member of the Krüppel-like transcription factor family containing a zinc finger domain. A previous study indicated that KLF5 is highly expressed in fetal blood vessels but dramatically decreased in adult vessels. ${ }^{27}$ Our results showed that KLF5 expression was elevated in the aortic tunicae media of TAD patients and was mostly located in the nucleus of HASMCs compared with normal aorta. The biological functions of KLF5 on the regulation of phenotype switch in vascular smooth muscle cells have been reported in several studies. ${ }^{22,23}$ The rescue experiments results in our study further indicate that KLF5 mediated the promoting effects of Oct 4 on the dedifferentiation in HASMCs. Interestingly, some studies reported that depletion of KLF5 in mouse embryos resulted in the reduction of Oct4, suggesting that KLF5 could also regulate the expression of Oct $4 .{ }^{28,29}$ These results indicate the probability of the existence of a positive feedback loop between KLF5 and Oct4 in HASMCs. Further studies are needed to investigate the function of this transcriptional feedback loop on the phenotypic switching of HASMCs.

In summary, the current study demonstrated that Oct4, which increased in the aortic media and primary HASMCs from TAD patients, promoted the phenotype switch of HASMCs by directly upregulating KLF5 expression (Figure 7). Future studies with appropriate animal models are needed to further confirm the conclusions of our present study.

The primary limitation of our study was that there was no elucidation of the mechanisms of Oct 4 activation in HASMCs in the pathogenesis of TAD. Many factors, such as transposition of the Oct4 gene ${ }^{30}$ and some environmental stimuli such as hypoxia, ${ }^{14}$ may all be potential causes of the activation of Oct4 expression in HASMCs via some specific mechanisms. Other limitations of our study were the relatively small number of samples and the heterogeneity of patients in the TAD and control groups, which might affect the comparability between the 2 groups. We mainly focused on the role of Oct4 and its regulative function on KLF5 during the phenotypic switching of HASMCs. The overall role of Oct4 in TAD remains to be explored.

\section{Conflict of Interest Statement}

Authors have nothing to disclose with regard to commercial support.

\section{References}

1. Howard DP, Banerjee A, Fairhead JF, Perkins J, Silver LE, Rothwell PM, et al. Population-based study of incidence and outcome of acute aortic dissection and premorbid risk factor control: 10-year results from the Oxford Vascular Study. Circulation. 2013;127:2031-7.

2. Di Bartolomeo R, Pantaleo A, Berretta P, Murana G, Castrovinci S, Cefarelli M, et al. Frozen elephant trunk surgery in acute aortic dissection. J Thorac Cardiovasc Surg. 2015;149:S105-9.

3. Vallabhajosyula P, Gottret JP, Bavaria JE, Desai ND, Szeto WY. Endovascular repair of the ascending aorta in patients at high risk for open repair. J Thorac Cardiovasc Surg. 2015;149:S144-50.

4. Barbour JR, Spinale FG, Ikonomidis JS. Proteinase systems and thoracic aortic aneurysm progression. J Surg Res. 2007;139:292-307. 
5. Wang X, LeMaire SA, Chen L, Shen YH, Gan Y, Bartsch H, et al. Increased collagen deposition and elevated expression of connective tissue growth factor in human thoracic aortic dissection. Circulation. 2006;114:I200-5.

6. Inamoto S, Kwartler CS, Lafont AL, Liang YY, Fadulu VT, Duraisamy S, et al. TGFBR2 mutations alter smooth muscle cell phenotype and predispose to thoracic aortic aneurysms and dissections. Cardiovasc Res. 2010;88:520-9.

7. Jones JA, Beck C, Barbour JR, Zavadzkas JA, Mukherjee R, Spinale FG, et al. Alterations in aortic cellular constituents during thoracic aortic aneurysm development: myofibroblast-mediated vascular remodeling. Am J Pathol. 2009;175: 1746-56.

8. Davis-Dusenbery BN, Wu C, Hata A. Micromanaging vascular smooth muscle cell differentiation and phenotypic modulation. Arterioscler Thromb Vasc Biol. 2011:31:2370-7.

9. Salmon M, Johnston WF, Woo A, Pope NH, Su G, Upchurch GR Jr, et al. KLF4 regulates abdominal aortic aneurysm morphology and deletion attenuates aneurysm formation. Circulation. 2013;128:S163-74.

10. Ailawadi G, Moehle CW, Pei H, Walton SP, Yang Z, Kron IL, et al. Smooth muscle phenotypic modulation is an early event in aortic aneurysms. $J$ Thorac Cardiovasc Surg. 2009;138:1392-9.

11. Atlasi Y, Mowla SJ, Ziaee SA, Bahrami AR. OCT-4, an embryonic stem cell marker, is highly expressed in bladder cancer. Int J Cancer. 2007;120:1598-602.

12. Takahashi K, Yamanaka S. Induction of pluripotent stem cells from mouse embryonic and adult fibroblast cultures by defined factors. Cell. 2006;126: 663-76.

13. Chiou SH, Wang ML, Chou YT, Chen CJ, Hong CF, Hsieh WJ, et al. Coexpression of Oct4 and Nanog enhances malignancy in lung adenocarcinoma by inducing cancer stem cell-like properties and epithelial-mesenchymal transdifferentiation. Cancer Res. 2010;70:10433-44.

14. Firth AL, Yao W, Remillard CV, Ogawa A, Yuan JX. Upregulation of Oct-4 isoforms in pulmonary artery smooth muscle cells from patients with pulmonary arterial hypertension. Am J Physiol Lung Cell Mol Physiol. 2010;298: L548-57.

15. Zhang XW, Zhang BY, Wang SW, Gong DJ, Han L, Xu ZY, et al. Twist-related protein 1 negatively regulated osteoblastic transdifferentiation of human aortic valve interstitial cells by directly inhibiting runt-related transcription factor 2 . J Thorac Cardiovasc Surg. 2014;148:1700-8.e1701.

16. Mueller T, Luetzkendorf J, Nerger K, Schmoll HJ, Mueller LP. Analysis of OCT4 expression in an extended panel of human tumor cell lines from multiple entities and in human mesenchymal stem cells. Cell Mol Life Sci CMLS. 2009;66: 495-503.

17. Wang X, Zhao Y, Xiao Z, Chen B, Wei Z, Wang B, et al. Alternative translation of OCT4 by an internal ribosome entry site and its novel function in stress response. Stem Cells. 2009;27:1265-75.

18. Hayashi K, Takahashi M, Nishida W, Yoshida K, Ohkawa Y, Kitabatake A, et al, Phenotypic modulation of vascular smooth muscle cells induced by unsaturated lysophosphatidic acids. Circ Res. 2001;89:251-8.
19. Christen T, Bochaton-Piallat ML, Neuville P, Rensen S, Redard M, van Eys G, et al. Cultured porcine coronary artery smooth muscle cells. A new model with advanced differentiation. Circ Res. 1999;85:99-107.

20. Sharov AA, Masui S, Sharova LV, Piao Y, Aiba K, Matoba R, et al. Iden tification of Pou5f1, Sox2, and Nanog downstream target genes with statistical confidence by applying a novel algorithm to time course microarray and genome-wide chromatin immunoprecipitation data. BMC Genomics 2008;9:269.

21. Boyer LA, Lee TI, Cole MF, Johnstone SE, Levine SS, Zucker JP, et al. Core tran scriptional regulatory circuitry in human embryonic stem cells. Cell. 2005;122: 947-56.

22. Kim SH, Yun SJ, Kim YH, Ha JM, Jin SY, Lee HS, et al. Essential role of kruppellike factor 5 during tumor necrosis factor alpha-induced phenotypic conversion of vascular smooth muscle cells. Biochem Biophys Res Commun. 2015;463: $1323-7$.

23. Zhang J, Zheng B, Zhou PP, Zhang RN, He M, Yang Z, et al. Vascular calcification is coupled with phenotypic conversion of vascular smooth muscle cells through Klf5-mediated transactivation of the Runx2 promoter. Biosci Rep. 2014;34:e00148.

24. Gonzalez RF, Allen L, Dobbs LG. Rat alveolar type I cells proliferate, express OCT-4, and exhibit phenotypic plasticity in vitro. Am J Physiol Lung Cell Mol Physiol. 2009;297:L1045-55.

25. Xie C, Ritchie RP, Huang H, Zhang J, Chen YE. Smooth muscle cell differentiation in vitro: models and underlying molecular mechanisms. Arterioscler Thromb Vasc Biol. 2011;31:1485-94.

26. Wang L, Zhang J, Fu W, Guo D, Jiang J, Wang Y. Association of smooth muscle cell phenotypes with extracellular matrix disorders in thoracic aortic dissection. $J$ Vasc Surg. 2012;56:1698-709. 1709.e1691.

27. Hoshino Y, Kurabayashi M, Kanda T, Hasegawa A, Sakamoto H, Okamoto E, et al. Regulated expression of the BTEB2 transcription factor in vascular smooth muscle cells: analysis of developmental and pathological expression profiles shows implications as a predictive factor for restenosis. Circulation. 2000;102: 2528-34.

28. Lin SC, Wani MA, Whitsett JA, Wells JM. Klf5 regulates lineage formation in the pre-implantation mouse embryo. Development. 2010;137:3953-63.

29. Parisi S, Passaro F, Aloia L, Manabe I, Nagai R, Pastore L, et al. Klf5 is involved in self-renewal of mouse embryonic stem cells. J Cell Sci. 2008;121: 2629-34.

30. Yamaguchi S, Yamazaki Y, Ishikawa Y, Kawaguchi N, Mukai H, Nakamura T EWSR1 is fused to POU5F1 in a bone tumor with translocation t(6;22)(p21;q12). Genes Chromosomes Cancer. 2005;43:217-22.

Key Words: thoracic aortic dissection, Oct4, KLF5, human aortic smooth muscle cells, phenotype transition 


\section{APPENDIX E1}

\section{Luciferase Reporter Assay}

The promotor sequence of KLF5 was amplified by PCR using the following primers: $5^{\prime}$-CCG CTC GAG AAT CCT GAA GGA GTG GGA AAA ACC- $3^{\prime}$ and $5^{\prime}$-CCC AAG CTT GTT TCG GGT GGA CTC CTC AGA C- $3^{\prime}$, where the underlined sequences are restriction enzyme sites for KpnI and HindIII, respectively. The mutant promotor sequence of KLF5 was generated by PCR using the following primers: $5^{\prime}$-CCG CTC GAG AAT CCT GAA GGA GTG GGA AAA ACC CAG GCT TTT ATG CAA GGA TCT GAA AAA ACG CTT GCC ATC AAA TCT TTT ATT CAG GCA TTC TGA GTA CTT CTT GT-3' and $5^{\prime}$-CCC AAG CTT GTT TCG GGT GGA CTC CTC AGA C-3', where the underlined sequence represents the mutated Oct 4 binding site. The PCR products were cloned into pGL3-basic vector upstream of the luciferase gene to construct pGL3-Pro-WT-KLF5 and pGL3-Pro-MT-KLF5, respectively.

Overexpression plasmid pcDNA-Oct4 was constructed by PCR cloning of human genomic DNA using the following primers: $5^{\prime}$-GCT CTA GAG CCA CCA TGG CGG GAC ACC TGG CTT-3' and 5'-CGG GAT CCT CAG TTT GAA TGC ATG-3', where the underlined sequences indicate the restriction enzyme sites for XbaI and BamHI, respectively. The PCR products were then cloned into pcDNAEF1 vector. Two hundred ninety-three cells were plated in 96-well plates $\left(5 \times 10^{3}\right.$ cells/well $)$ and co-transfected in triplicate with reporter plasmid (pGL3-Pro-WT-KLF5 or pGL3-Pro-MT-KLF5), pcDNAOct4, and an internal control plasmid pGL-TK using Lipofectamine 2000 (Invitrogen). After 48 hours of transfection, luciferase activities were measured using the Dual Luciferase Reporter Assay system (Promega E1980, Madison, Wis). Relative promoter activities are expressed as luminescence relative units normalized to Renilla luciferase activity expression in the cell extracts.

\section{ChIP}

The ChIP assay was performed using the EZ-ChIP Chromatin Immunoprecipitation Kit (EMD Millipore, Billerica, Mass) according to the manufacturer's instruction. HASMCs were transfected with Ad.Oct4. After 48 hours, HASMCs were fixed using $1 \%$ formaldehyde, sonicated using VCX400 ultrasonic processor (Sonics \& Materials, Newtown, Conn), and immunoprecipitated with $3 \mu \mathrm{g}$ of anti-Oct4 antibody-ChIP grade (ab19857; Abcam) or normal rabbit IgG. The primer of the binding site in the KLF5 promoter was forward, 5'-GGA TGG GAA GTA GAA ATG-3'; reverse, 5'-TGA GTG TAT CCG TTT AAC AA- $3^{\prime}$.

\section{Semiquantitation of Immunofluorescent Image}

ImageJ software (NIH, Bethesda, Md) was used to measure the intensity of staining. Fluorescence intensity of Oct 4 was represented as the ratio of the integrated density of red fluorescence to the area of the cell nucleus. 
TABLE E1. Patient characteristics

\begin{tabular}{lcc}
\hline \multicolumn{1}{c}{ Variables } & $\begin{array}{c}\text { TAD } \\
(\mathbf{n = 1 2 )}\end{array}$ & $\begin{array}{c}\text { Controls } \\
(\mathbf{n = 6}=\mathbf{6})\end{array}$ \\
\hline Age (y), mean \pm SD & $50.3 \pm 8.3$ & $44.3 \pm 9.0$ \\
Male, n (\%) & $9(75.0)$ & $4(66.7)$ \\
Hypertension, n (\%) & $8(66.7)$ & $1(16.7)$ \\
Diabetes mellitus, $\mathrm{n}(\%)$ & $2(16.7)$ & $1(16.7)$ \\
Taking anti-lipid medication, n (\%) & $4(33.3)$ & 0 \\
History of cerebrovascular disease, $\mathrm{n}(\%)$ & $2(16.7)$ & 0 \\
Aortic diameter at sample site $(\mathrm{cm})$, & $5.7 \pm 1.0$ & $\mathrm{NA}$ \\
$\quad$ mean \pm SD & & \\
\hline TAD, Thoracic aortic dissection; $S D$, standard deviation; $N A$, not available.
\end{tabular}

TABLE E2. Primers for qRT-PCR

\begin{tabular}{|c|c|c|}
\hline Gene & Forward primer & Reverse primer \\
\hline$\alpha$-SMA & 5'-GTGTTGCCCCTGAAGAGCAT-3' & $5^{\prime}$-GCTGGGACATTGAAAGTCTCA-3' \\
\hline MYH11 & $5^{\prime}$ - CGCCAAGAGACTCGTCTGG-3' & 5'-TCTTTCCCAACCGTGACCTTC-3' \\
\hline KLF5 & $5^{\prime}$-CCTGGTCCAGACAAGATGTGA-3' & 5'-GAACTGGTCTACGACTGAGGC-3' \\
\hline Oct4-1 & 5'-GTGAGAGGCAACCTGGAGAATT-3' & 5'-CCTCAGTTTGAATGCATGGGAG-3' \\
\hline Oct $4 \mathrm{~A}-1$ & $5^{\prime}-$ CCCCTGGTGCCGTGAA- $3^{\prime}$ & 5'-GCAAATTGCTCGAGTTCTTTCTG-3' \\
\hline Oct $4 \mathrm{~A}-2$ & $5^{\prime}$-CGTGAAGCTGGAGAAGGAGAAGCTG-3' & $5^{\prime}$-CAAGGGCCGCAGCTTACACATGTTC-3' \\
\hline Oct4-2 & $5^{\prime}$-GACAACAATGAAAATCTTCAGGAGA- $3^{\prime}$ & 5'-TTCTGGCGCCGGTTACAGAACCA-3' \\
\hline Nanog & 5'-TTTGTGGGCCTGAAGAAAACT-3' & 5'-AGGGCTGTCCTGAATAAGCAG-3' \\
\hline SOX2 & 5'-TGGACAGTTACGCGCACAT-3' & 5'-CGAGTAGGACATGCTGTAGGT-3' \\
\hline TCF3 & 5'-ACGAGCGTATGGGCTACCA-3' & 5'-GTTATTGCTTGAGTGATCCGGG-3' \\
\hline BMP4 & $5^{\prime}$-AAAGTCGCCGAGATTCAGGG-3' & 5'-GACGGCACTCTTGCTAGGC-3' \\
\hline Notch1 & 5'-GAGGCGTGGCAGACTATGC-3' & $5^{\prime}$-CTTGTACTCCGTCAGCGTGA-3' \\
\hline DUSP27 & 5'-GCCAGTATTCGATGGTCTCAG-3' & 5'-CCCGGTGGCTTGAGTTCTTC-3' \\
\hline DUSP10 & 5'-ATCGGCTACGTCATCAACGTC-3' & 5'-TCATCCGAGTGTGCTTCATCA-3' \\
\hline Lefty 1 & 5'-GGGCGACTGTGACCCTGAAGCA-3' & 5'-TTGGCACGAGCGCACCATCC-3' \\
\hline Foxd3 & 5'-GACGACGGGCTGGAAGAGAA-3' & 5'-GCCTCCTTGGGCAATGTCA-3' \\
\hline Msc & 5'-CCCCGACACTAAGCTCTCCA-3' & 5'-GTAGCCGTTCTCATAGCGGT-3' \\
\hline TDGF1 & 5'-TTTGAACTGGGATTAGTTGCCG-3' & 5'-GGGGCCAAATGCTGTCATCT-3' \\
\hline Nodal & 5'-CTGCTTAGAGCGGTTTCAGATG-3' & 5'-CGAGAGGTTGGAGTAGAGCATAA-3' \\
\hline Sall4 & 5'-AGCACATCAACTCGGAGGAG-3' & 5'-CATTCCCTGGGTGGTTCACTG-3' \\
\hline Etv5 & 5'-TCAGCAAGTCCCTTTTATGGTC- ${ }^{\prime}$ & 5'-GCTCTTCAGAATCGTGAGCCA-3' \\
\hline Sfrp1 & 5'-ACGTGGGCTACAAGAAGATGG-3' & 5'-CAGCGACACGGGTAGATGG-3' \\
\hline Myc & 5'-GGCTCCTGGCAAAAGGTCA-3' & 5'-CTGCGTAGTTGTGCTGATGT-3' \\
\hline Dppa3 & 5'-TTAATCCAACCTACATCCCAGGG-3' & 5'-AGGGGAAACAGATTCGCTACTA-3' \\
\hline MMP2 & 5'-CCACTGCCTTCGATACAC- $3^{\prime}$ & 5'-GAGCCACTCTCTGGAATCTTAAA-3' \\
\hline MMP9 & 5'-TGTACCGCTATGGTTACACTCG-3' & 5'-GGCAGGGACAGTTGCTTCT-3' \\
\hline GAPDH & $5^{\prime}$-CTGGGCTACACTGAGCACC-3' & 5'-AAGTGGTCGTTGAGGGCAAT-3' \\
\hline
\end{tabular}




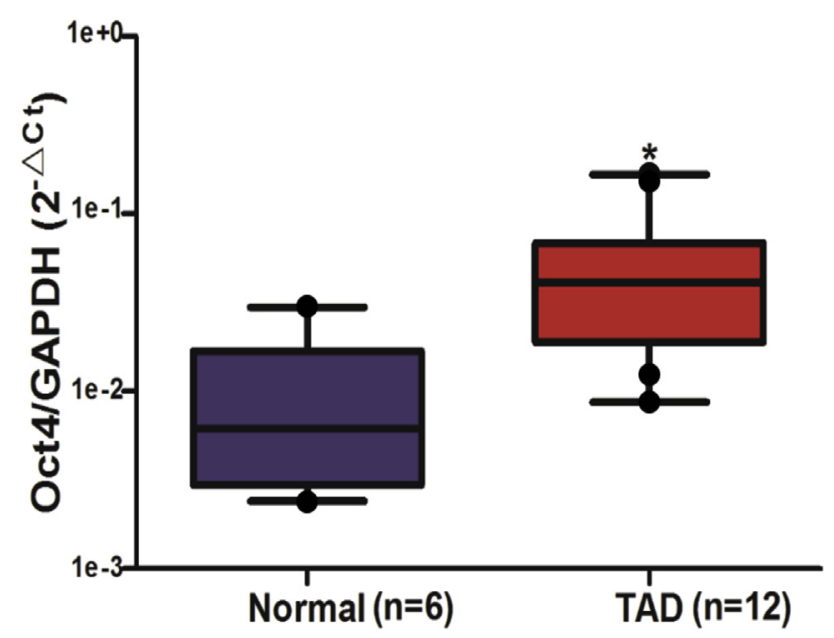

FIGURE E1. Increased Oct 4 mRNA in aortic tissues from TAD patients. qRT-PCR showed that the mRNA level of Oct 4 was significantly increased in the TAD group $(\mathrm{n}=12)$ compared with normal controls $(\mathrm{n}=6)$. $* P<.05$ versus controls. $T A D$, Thoracic aortic dissection; Oct4, octamer binding protein-4; GAPDH, glyceraldehyde-3-phosphate dehydrogenase.
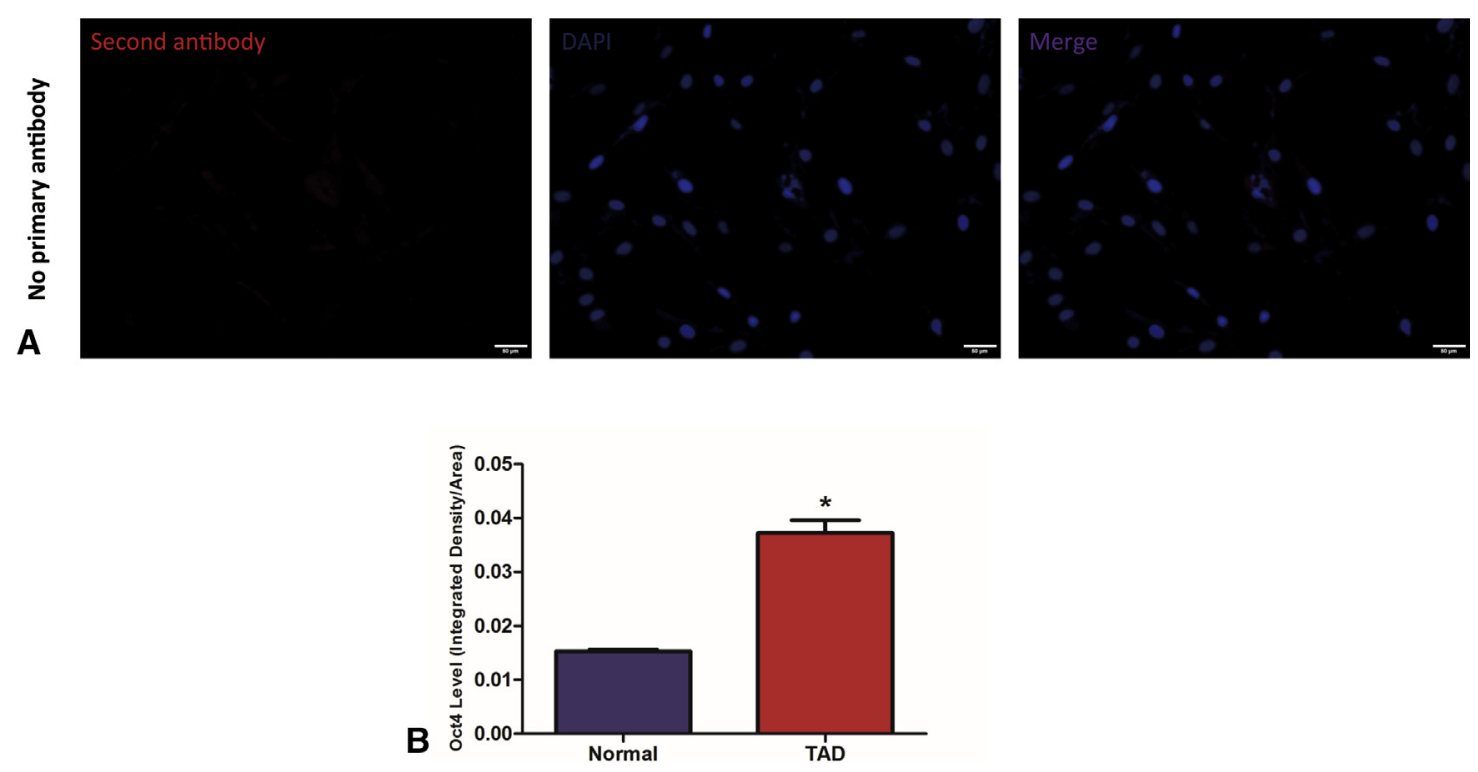

FIGURE E2. Negative control and semiquantitation of Oct4 immunofluorescent staining in HASMCs. A, Negative control without primary antibody demonstrates the specificity of Oct 4 staining. B, The averaged fluorescence intensity of Oct 4 staining. $* P<.05$ versus controls. Oct 4 , Octamer binding protein-4; $T A D$, thoracic aortic dissection. 


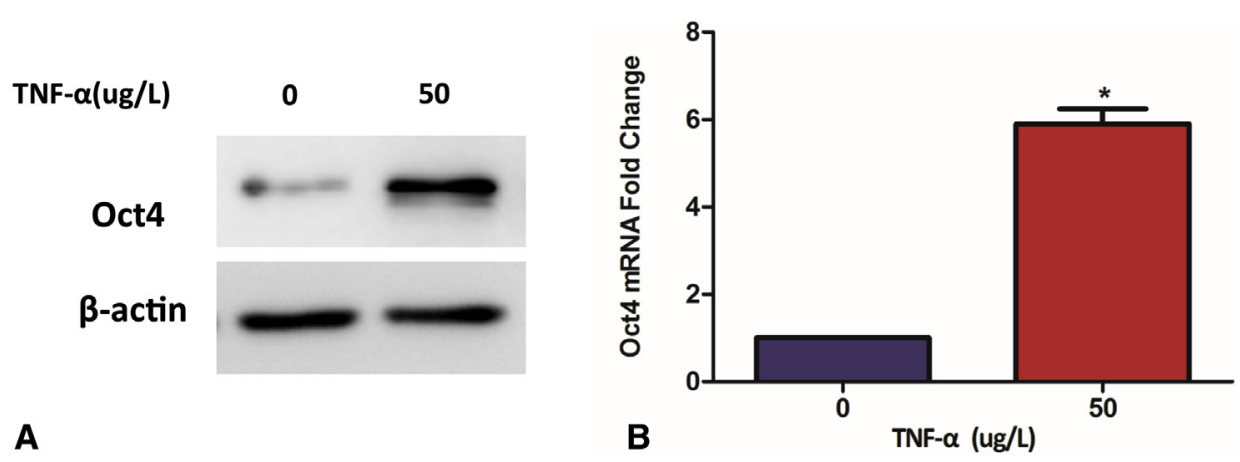

FIGURE E3. TNF- $\alpha$ induced the expression of Oct4 in HASMCs. A and B, Western blot and qRT-PCR showed that the expression of Oct4 was increased significantly in HASMCs stimulated by TNF- $\alpha(50 \mu \mathrm{g} / \mathrm{L})$ for 48 hours. TNF- $\alpha$, Tumor necrosis factor $\alpha$; Oct 4 , octamer binding protein- 4 .
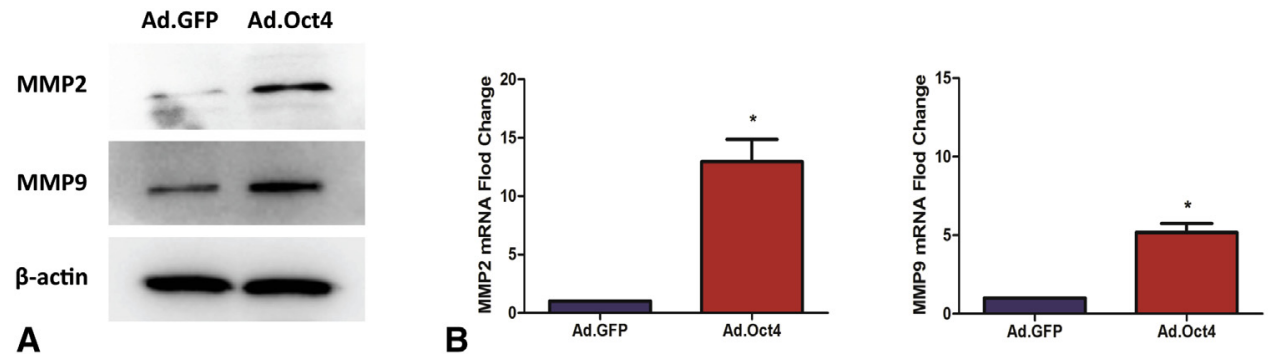

FIGURE E4. Oct4 upregulated the expression of MMP2 and MMP9 in HASMCS. A and B, Western blot and qRT-PCR showed that the expressions of MMP2 and MMP9 were significantly increased in the Ad.Oct4 group compared with the Ad.GFP group ( $\mathrm{n}=3$ for each cell group). $* P<.05$ versus Ad.GFP HASMCs. Ad.Oct4, Adenovirus overexpressing Oct4; Ad.GFP, adenovirus expressing GFP. 